\title{
„ÖSTERREICHISCHE“ UND ANDERE IRONIE IN DEN TEXTEN VON OLGA FLOR, TERESA PRÄAUER UND CORDULA SIMON
}

\begin{abstract}
Den Ausgangspunkt für den Artikel bildet die Aussage von Kathrin Röggla, derzufolge „Was in Österreich - nicht nur in der Literatur - Tradition hat, ist Ironie“. Den Gegenstand der Untersuchung bilden Ironie-Konzepte in drei Romanen der österreichischen Autorinnen der Gegenwart Olga Flor, Teresa Präauer, Cordula Simon. Die Fragestellung ist auf die spezifisch „österreichische“ Ironie ausgerichtet, die, so die These, eine besondere Form der Auseinandersetzung mit aktuellen sozialen und politischen Themenkomplexen darstellt.
\end{abstract}

SCHLÜSSELWÖRTER: Österreichische Gegenwartsliteratur, Ironie

\section{“AUSTRIAN" AND OTHER IRONY IN THE FICTION BY OLGA FLOR, TERESA PRÄAU- ER AND CORDULA SIMON}

AbSTRACT: "There is a tradition of irony in Austria, not only in literature", said Kathrin Röggla in an interview in 2012; my article considers the assertion as a starting point to an analysis of three novels by contemporary Austrian women authors Flor, Präauer and Simon. The aim is to examine the different patterns of irony by Flor, Präauer and Simon in order to assess what the "Austrian" concept of irony can mean today. Furthermore, I would like to consider the „Austrian“ irony as a specific Austrian cue to express involvement in current political matters.

KEYwORDS: contemporary Austrian novel, irony

Ich sage immer noch: Ich bin eine österreichische Autorin, der sprachkritischen und sprachspielerischen Tradition sehr verbunden. Da kommt aus Österreich viel, was mit mir zu tun hat. Vor allem der Umgang mit Ironie und Witz. In Deutschland ist die Literatur sehr aufklärerisch im klassischen Sinn. Das wäre mir zu naiv.

- so Kathrin Röggla in einem Interview für Die Presse 2012 (Gauhofer 2012). Und: „Was in Österreich - nicht nur in der Literatur - Tradition hat, ist Ironie." (Gauhofer 2012) Ich beginne mit diesen Aussagen, weil Röggla hier etwas formuliert, was als ein

Kalina Kupczyńska - Uniwersytet Łódzki, Łódź, kalina.kupczynska@uni.lodz.pl 
Konsens in der literatur- wie auch der kulturwissenschaftlichen Forschung zu Österreich gilt und was dennoch relativ schwer zu fassen ist. Nicht nur, weil die Reihe der österreichischen Ironie-Meister_innen lang und heterogen ist - von Musil über Torberg bis zu Schindel oder Vertlib, von Artmann über Jandl bis zu Jelinek, etc. Sondern, weil über Ironie zu sprechen ein „riskantes Unterfangen“ ist, ,risky business" ${ }^{\text {“1 }}$, wie Linda Hutcheon schreibt, weil die Ironie, so Wayne C. Booth, als „the mother of confusion“ (Booth 1975: ix) gilt. Für Hutcheon wie auch für Booth sind - bei der unterschiedlichen Fokussierung ihrer Ironie-Studien - die Unterhöhlung der Klarheit und die Dialektik die brisantesten Aspekte der Ironie (Booth 1975: ix; Hutcheon 1994: 27f.). In Rögglas Aussage werden Sprachspiel, Sprachkritik, Ironie und Witz zusammengedacht, in ihrem Vergleich zu Deutschland lässt sich eine subversive Note gegenüber klassischem aufklärerischem Ethos wahrnehmen und damit ein Verständnis von Ironie als einem „counter-discourse“ (Terdiman 1985), der ein Potential zur Herausforderung der Hierarchien birgt. Röggla blendet das Konzept der romantischen Ironie - das ja ebenfalls zur deutschen Literaturtradition gehört - aus, womit sich eine für die „österreichische“ Ironie wichtige Unterscheidung abzeichnet. Der Clou der „österreichischen“ Ironie liegt darin, anzuecken, d. h. Widersprüche sichtbar zu machen - Linda Hutcheon nutzt dafür das Wort „edge“. ${ }^{2}$ Was der ironischen Haltung zuweilen vorgeworfen wurde Distanzierung im negativen Sinn der Abgrenzung bzw. gar Überheblichkeit - kann ebenso positiv aufgefasst werden, und zwar als Aufzeigen von neuen Perspektiven, als eine differenzierte Sicht; ${ }^{3}$ auch dies ist für das Verständnis der „österreichischen“ Ironie wichtig. So lassen sich Ich in Gelb von Olga Flor (2015), Oh Schimmi von Teresa Präauer (2016) und in Wie man schlafen soll von Cordula Simon (2016) nicht trotz, sondern dank der dort präsenten Ironie als Beispiele der spezifisch österreichischen Form von sozialkritischer Literatur lesen. Wie ich zeigen möchte, vollzieht sich im Umgang mit der Tradition der Ironie in der österreichischen Literatur der Gegenwart eine besondere Art von littérature engagée und zugleich eine Meta-Reflexion über die modernen Ironie-Arten.

${ }^{1}$ Linda Hutcheon: „However you might choose to talk about the difference between irony that is seen to exclude and finalize and irony that is seen to relate and relativize, the politics of irony are never simple and never single. [...] More is at stake here [...] than may be the case with other discursive strategies, and that "more" has a lot to do with power. This is why the language used to talk about irony [...] is so often the language of risk: irony is "dangerous" and "tricky" (Lejeune 1989: 64) - for ironist, interpreter and target alike." Hutcheon, L. (1994). Risky business. London: Routledge, S. 17.

${ }^{2}$ Vgl. das Kapitel „The cutting edge“, Hutcheon, L. (1994). Risky business. London: Routledge, S. $37-56$.

${ }^{3}$ Vgl. Linda Hutcheon: „Distance can, of course, suggest the non-committal, the inferred refusal of engagement and involvement, and so its more pejorative associations are with indifference or even Olympian disdain and superiority. But distancing reserve can also be interpreted as a means to a new perspective from which things can be shown and thus seen differently [...]" (Hervorhebungen im Original). Hutcheon, L. (1994). Risky business. London: Routledge, S. 49 


\section{Konstruktive Ironie: Olga Flors Ich in Gelb}

„Ironie ist: einen Klerikalen so darstellen, daß neben ihm auch ein Bolschewik getroffen ist, einen Trottel so darstellen, daß der Autor fühlt: das bin ich ja zum Teil selbst.“ (Musil 1978: 1939) Musil formulierte eine Ironie-Konzeption, die er als „konstruktive Ironie“ bezeichnete - sie ergibt sich aus einem Zusammenhang der Dinge. ${ }^{4}$ Das Konstruktive in Musils Ironie lag in der „Schöpfung eines ironischen Stils, in dem die Wirklichkeit erforscht werden könne“ (Japp 1983: 318). Ironie zeigte sich „als Vorbehalt, die sich nicht nur gegen eine Sache richtet, sondern den Vorbehalt zu ihrer eigenen Sache macht und so die Sache allererst hervorbringt“"(Japp 1983: 319), daraus entsprang auch ihre Produktivität. Ein Bestandteil des so aufgefassten ironischen Stils in Der Mann ohne Eigenschaften ist eine „Interaktion zwischen verschiedenen Diskurstypen " - durch die Ironie erfolgt eine Kritik an Diskursen, die etabliertes diskursives Verhalten hinterfragt und Diskurse so für Schaffung neuer Diskursformationen öffnet. ${ }^{5}$

In Olga Flors Roman Ich in Gelb weiß das Model Alice viel: Sie kennt den Kommunikationscode und das Machtgefüge innerhalb der Modewelt, sie kennt die Gesetze der Netzwelt, sie führt einen Ego-Blog (ihr Nickname ist nextGirl). Damit wird schnell klar: Sie partizipiert an Diskursformationen, die um die Fragen der Identität kreisen, explizit um self-fashioning in der Mode und im Netz. Alice weiß allerdings zu viel, und wenn sie das Zuviel - Infos über die Ausbeutung blutjunger Models durch Modelagenturen auf ihrem Blog postet, folgen Drohungen: „Schadenersatzklage“, „Rufschädigung“, „Unterlassung unwahrer Behauptungen“(Flor 2015: 144). Ihre Antwort folgt schlagartig: „Die haben ja keine Ahnung, mit wem sie sich anlegen, diese Digital Naives: Wer sich ins Netz begibt, geht darin unter! [...] Wir starten eine Linklawine, Leute, postet, repostet, rerepostet und shitstormt, bis das wifi glüht! nG“ (Flor 2015: 144). Das Einzige, was Alice ernsthaft Sorgen macht, ist: „Die Vorstellung, sich nicht mehr öffentlich äußern zu können“ (Flor 2015: 142). Drei Wochen später postet Alice: „Bin mit Laura und Papa im Tiergarten gewesen [...]“, Bilder folgen. Stattdessen ein Nashorngedicht:

Wenn Nashornnasen schrecklich niesen

Kann Nashornhörner das verdrießen

Selbst dickste Häuter hängen drum

Im Winter lieber drinnen rum. (Flor 2015: 137)

${ }^{4}$ Vgl. Uwe Japp: „Diese Art von Ironie nannte Musil die ,konstruktive Ironie‘, und er war der Auffassung, daß sie im heutigen Deutschland ziemlich unbekannt sei. Es sei der Zusammenhang der Dinge, aus dem sie nackt hervorgehe.“ Japp, U. (1983). Theorie der Ironie. Frankfurt a. M.: Klostermann, S. 318.

${ }^{5}$ Walter Moser: ,[...] irony is an agent in a critique of discourse which is used to question established discursive behavior, attitudes, positions and beliefs, and to open them up to the possibility of new formations of discourse." Moser, W. (1984). The Factual in Fiction: The Case of Robert Musil. Poetics Today, vol. 5, no. 2, S. 411-428, hier S. 414. 
Ein früherer Eintrag enthält eine Aufzählung: „Hosenanzug, samtig; Schneckenhauspullover, Gummirosenrock, Schuppenstretch, Farbeinkehr, spiegelbesetzt; hochfliegendes Sommerkleid [...] plus Moonboots.“ (Flor 2015: 138) Alice gibt sich wissend: „Was ich heute nicht weiß, ist morgen schon überholt, wozu also noch wissen wollen.“ (Flor 2015: 17) „Aus geprüften, abgelagerten und vernetzten Bildern kann ich mir die Welt zusammenbauen. Dabei muss ständig neues Beweismaterial hochgeladen werden, damit man mit genau diesem Jetzt Schritt halten kann.“ (Flor 2015: 28) „Styling, ist überhaupt nichts als Styling. Selbstbild vs. Fremdbild. Gibt es das überhaupt, Fremdbild?" (Flor 2015: 200)

Flor wählte für den Roman die Form des Blogs, eine Form, die Heterogenität der diskursiven Ebenen wie auch des Bildmaterials begrüßt, und zugleich ein postmodernes digitales Format bietet, das Wissen - meistens subjektives, privates Wissen - vermittelt. Blogs funktionieren in einem Medium, in dem ,sich Realität und Fiktion zum Verwechseln mischen"(Herbst 2005: 14), Alban Nikolai Herbst bezeichnete sie einmal als „eine interaktive Bühne“, auf der sich in Quasi-Oralität ein „Tanz der Ich-Ideale“ (Herbst 2005: 15) abspielt. Die im Zusammenhang mit Musil erwähnte „Interaktion zwischen verschiedenen Diskurstypen", die in seinem Roman die ironische Komponente generiert, wirkt hier alles andere als überraschend, denn, „Weblogs sind grundsätzlich brikoliert" (Herbst 2005: 24), was der zeitgenössischen Leserin durchaus vertraut sein könnte. Die Produktivität der diskursiven Interaktion wird allerdings erst dann plausibel, wenn man bedenkt, dass die Bloggerin dreizehn ist, und dennoch die Diskurse soweit beherrscht, dass sie souverän im Cyberraum agieren kann, obwohl (oder: weil?) sie sich zuweilen kindisch gibt. Ironisch ist nicht die Figur selbst, sondern, wie bei Musil, der Zusammenhang der Dinge, der hier hergestellt wird. Also: Ein dreizehnjähriges Model postet sein Wissen über die Wirklichkeit, das auf die Überzeugung gründet, diese Wirklichkeit könne sie sich im Internet „festschreiben“, ja „sich in ihr verankern“ (Flor 2015: 29). „Nur ein dokumentiertes Leben ist ein echtes“ (Flor 2015: 48), schreibt Alice. Und: „Was einmal im Internet drin ist, geht nicht mehr raus, garantiert washing-proof.“ (Flor 2015: 192)

Die Ironie in Flors Konstruktion der emphatisch über die „digitale Verankerung“ postenden Bloggerin ist erhellend, vor allem wenn man bedenkt, dass der Glaube an eine digitale Festschreibung paradoxerweise umso fester wird, je heftiger seine Grundlagen erschüttert werden. Und diese werden - zumindest rechtmäßig - in der Tat erschüttert: Im Mai 2014 hat der Europäische Gerichtshof beschlossen, dass „der Betreiber der Suchmaschine Google unter bestimmten Umständen Verweise auf personenbezogene Daten aus seinem Index streichen muss" ${ }^{6}$ In ihrer Reaktion auf diesen Beschluss wies Aleida Assmann auf den Unterschied zwischen Speichern und Erinnern hin - was von

${ }^{6}$ Löschungsfrage gegen den Suchmaschinenbetreiber. Abgerufen am 9.11.2018 von https://www.dsb. gv.at/fragen-und-antworten. 
Google gelöscht wird, muss nicht zwangsläufig vergessen werden (Martens 2015). Und dennoch - Flor berührt eine wunde Stelle der digitalen Identität, denn sie provoziert Fragen: Ist nun eine ,digitale Verankerung“ möglich oder ist sie wirklich? In welcher Fiktion befindet sich die Leserin von Ich in Gelb - in der Roman-Fiktion oder in der Internet-Fiktion? Oder in beiden?

Flor entwickelt ihre Mode-Bloggerin wie ein Musterbeispiel (oder auch: Modell) des Paradigmas, das Andrew Hoskins als „,connective turn“ (Hoskins 2011: 270) formulierte - Alice praktiziert das für die ,partizipatorische“ digitale Medienkultur typische ,sharing without sharing“ (Hoskins 2017: 2), spiegelt sich narzisstisch in ihren Blog-Einträgen, und damit hält sie auch der Leserin den Spiegel vor, denn, wie Hoskins in Digital Memory and Media schreibt: „We are already all addicts.“ (Hoskins 2017: 3) So auch Aleida Assmann ex negativo über diese Funktion des Mediums Internet: „Es bietet keine Vision einer besseren Welt, sondern ist ein drastischer Spiegel der Welt wie sie ist und wie sie sich rapide verändert mit all ihren Idealen, Ideen, Chancen, Fehlern und Gefahren.“ (Assmann 2018: 129)

Ein ausgeklügelter ironischer Twist besteht außerdem darin, dass die Form des Romans der Zeitstruktur eines Blogs folgt, d. h. die Leserin mit den neuesten Einträgen beginnt und in Alices Vergangenheit einzutauchen scheint, was aber eine Täuschung ist, weil Blogs Vergangenes eigentlich nicht kennen: „Ist ja immer heute. Wo auch immer das ist, hier und jetzt.“ (Flor 2015: 102) Auch Zeitempfinden verändert sich im Cyberraum, das physikalische Gesetz der Zeitumkehrinvarianz wird herausgefordert. ${ }^{7}$ Für den mächtigen Sog des Netzes (und der Mode) findet sich im Roman eine mythische Figuration: Bilder der Medusa-Köpfe geistern durch den Roman, Alice nennt explizit den versteinernden Blick der Gorgone (Flor 2015: 124). Die ironischen Aspekte der komplexen Text-Bild-Struktur des Romans erkennt man in der Parallelisierung von der Schreckensgestalt der mythischen Medusa mit der scheinbar verspielten und lebensbejahenden Modewelt - Hüte à la Medusenhaupt wechseln sich mit antiken Medusendarstellungen ab (Abb. 1 und 2). Zusätzlich stößt man auf Bilder, die Assoziationen zwischen Meduse und realen (Meeres)Tieren suggerieren: Oktopussen, Quallen, Würmern (Abb. 3 und 4). Dabei ist Bianka, die die Blog-Einträge von nextGirl kommentiert, Wirtin eines Bandwurms, den sie in einer fantastisch-morbiden Szene während einer penibel inszenierten Mode-Show, in ei-

\footnotetext{
7 „Zeitumkehrinvarianz“ heißt auch das Password für den Blogroman, wie man im Anhang des Romans lesen kann. Vgl. Olga Flor: „Dieser Begriff bedeutet, dass bestimmte physikalische Gleichungen invariant gegenüber der Zeitumkehr sind, sich also nicht verändern, wenn die Zeit durch die negative Zeit ersetzt wird, die Zeit also in die umgekehrte Richtung liefe, was natürlich praktisch nicht möglich ist. Es ist ein reines Konstrukt, das hilft, zu untersuchen, ob physikalische Vorgänge auch in umgekehrter Richtung ablaufen können.“ Flor, O. (23. Juni 2015) im Gespräch mit Katrin Hillgruber. Wer sagt hier „Ich“ und in welcher Absicht? Abgerufen von http://volltext.net/texte/wer-sagt-hier-ich-und-in-welcher-absicht/.
} 


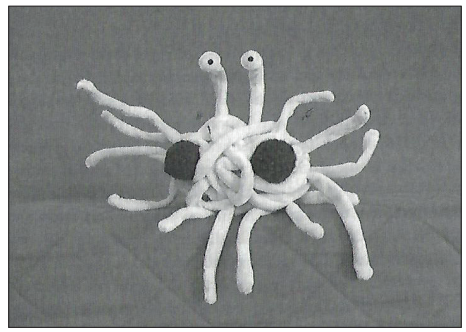

Abb. 1.: Ich in Gelb, S. 59.

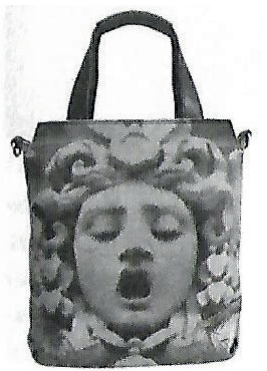

Abb. 2.: Ich in Gelb, S. 31.

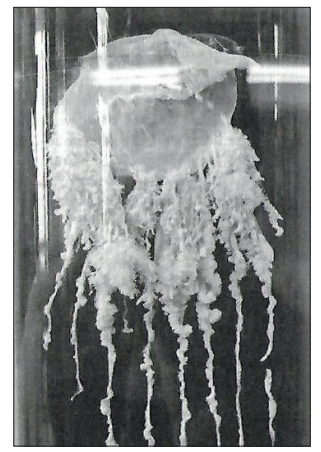

Abb. 3.: Ich in Gelb, S. 41.

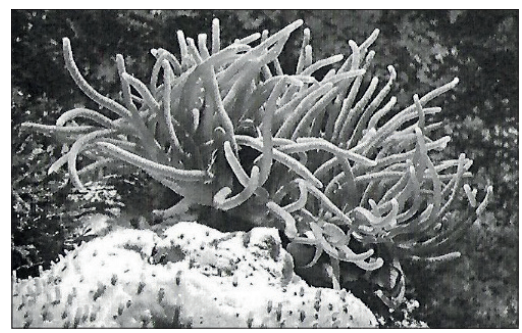

Abb. 4.: Ich in Gelb, S. 125. 
ner riesigen Wasserblase schwimmend, erbricht. ${ }^{8}$ „Die Faszination des Monströsen“ (Hillgruber 2015) bietet eine andere ironische Note, wenn man bedenkt, dass der Parasit Biancas Erfolg in der Modebranche sichert und sie glücklich macht: „Ich gefalle mir. Zum ersten Mal seit Langem gefalle ich mir selbst.“ (Flor 2015: 197) Die ambivalente Erscheinung der mythischen Medusa wird zur Personifikation der Modewelt, deren Kulissen hier punktuell entlarvt werden; Monstrosität und Schönheit, Glamour und Dreck, Ich-Apotheose und Selbstzerstörung liegen so nah beieinander, dass sie ohne die ironische Ebene der selbstgefälligen Blog-Plapperei der dreizehnjährigen nextGirl in neobarockes Pathos ausarten würden. Denn: Wo das Medusenhaupt auftaucht, lauert der Tod - der Tod durch Erstarren, den der bloße Anblick des Monstrums erzeugt, oder auch, bei Freud, der symbolische Tod des Mannes, denn „(d)er Schreck der Meduse ist [...] Kastrationsschreck, der an einen Anblick geknüpft ist.“" (Freud 1940: 273) Die Faszination der nextGirl für die Medusa wäre also auch als eine Machtphantasie zu deuten, was im Modeuniversum, in dem Modedesigner symbolisch über Geburten und Tode der Noch-nicht-Frauen entscheiden, plausibel erscheint (Rohde-Dachser 1991: 133).

\section{Pop-Ironie? Teresa Präauers Oh Schimmi}

Die Verschwisterung des Ironie-Diskurses mit dem Pop-Diskurs wird in den 1990er Jahren vor allem im Zusammenhang mit der Sprache der Pop-Romane (von Florian Illies, Benjamin von Stuckrad-Barre, Christian Kracht) und der dort herausgestellten Überaffirmation des Bestehenden evident. In der Literatur wird die Oberflächenästhetik des Pop über Verfahren der Auflistung, der Nennung der Markennamen, der Beschreibung der Wirklichkeit als einer Kulturenzyklopädie erkennbar, welche Eingeweihten stichwortartig ein Gefühl von Vertrautheit vermitteln sollte (was den Pop-Autoren im Feuilleton den Vorwurf eines „Geschmacksterrorismus“ einbrachte) (Baßler 2005: 107). Die deutschen Pop-Romane - Generation Golf, Soloalbum, Faserland - haben bekanntlich einen eher wenig aufregenden Plot, in dem ein homodiegetischer Ich-Erzähler einkauft, isst, reist, auf Partys geht, fernsieht, etc. Der Pop-Effekt ergibt sich aus einer besonderen sprachlichen Konstruktion - nicht anders ist es im Fall von Teresa Präauers Oh Schimmi von 2016. Aus der Diegese zu schließen wäre es ein sozialkritischer Roman: Ein Teenager lebt mit seiner depressiven Mutter in einem Großstadtapartment, der Vater hat sie verlassen, um Krisengebiete zu „,emerging markets“ (Präauer 2016: 80) zu erklären, das Leben des Jungen besteht aus Fernsehen und gelegentlichen

8 „Der Menschenfischleib explodierte, so sah das aus, der Bauch schwoll und zog sich zusammen, der ganze Körper krampfte, der Mund wurde aufgerissen und erbrach eine dunkle Substanz, stoßweise, und dieses Dunkle begann sich bandartig zu winden, dann half die Fischfrau nach und zog und zog, als hätte sie ein Wollknäuel verschluckt, und schließlich löste sich das Ding von ihr und schwamm davon, so weit es eben ging.“ (Flor 2015: 205) 
Ausbrüchen aus dem Wohnturm in das brodelnde Stadtleben. Jimmy wurde als Kind von einem Rodeo-Pferd umgerannt und gilt seitdem als Person mit „,besonderen Bedürfnissen" (Präauer 2016: 65), ist dementsprechend einsam; Trost sucht und findet er bei der mexikanischen Putzfrau Guadalupe, bei Tierfilmen sowie bei Sex-Telefon. Wenn er nicht wie die Figuren bei Illies und Kohorten von Ausflügen zu Ikea bzw. auf Sylt, von Barbour Jacken sowie von gelegentlichem Liebeskummer erzählt, dann wohl deswegen, weil er eben anders ist. „Abhängig“ (Präauer 2016: 89) haben ihn einmal die Nachbarn genannt, was Schimmi als Beschreibungskategorie beunruhigt, weil er sich vollkommen anders wahrnimmt. „Ich bin pan-animalisch“ (Präauer 2016: 94), sagt Schimmi, „der schwarze Gorilla, das bin ich“ (Präauer 2016: 117), „Krönung der Schöpfung“, „König der Tiere“ (Präauer 2016: 131).

Der unterkühlte Ton der Pop-Romane findet sich hier kaum, die Sprache des Romans ist durch Schimmis begeisterte, wirre Triumphreden aufgeheizt. Das Präsens von Schimmis Rede erinnert zuweilen entfernt an das schnelle Realzeit-Stakkato eines Rainald Goetz. Schimmi bekommt ab und zu mit, wie ihn die anderen wahrnehmen „bloß ein kleiner Junge“ (Präauer 2016: 15), ein Barmann verkauft ihm kein Bier ohne Ausweis - aber er lässt sich dadurch in seiner animalischen Überlegenheit nicht stören. So betritt er eine Disko:

Hier kommt der Hotteste unter den Hotten, der Fresheste unter den Freshen, der Obermakake, der affengeile. Er ist unser Vortänzer, / er ist der Aufreißer. / Er hat die Hosen an, / nur er weiß, wie man rappen kann. / Er ist der Styler. / kein Blender, ein Keiler. / Er trägt seine Snapback, Jungle Fever, / Rat Pack, Ape Swag. (Präauer 2016: 127)

Von wegen Barbour Jacke und Ikea, Spex und Ralph-Laurent-Hemden - Schimmis Selbstbeschreibung schaukelt sich an Gadgets und Songtexten der US-amerikanischen Hip-Hopper und Rapper hoch: „Kanye, ich kenn dich, Jay-Z, auf dich steh ich“ (Präauer 2016: 129). Ninni, sein Traum-,,Girl“ trägt blonde Polyester-Strähnen im Haar und goldene Kunstnägel „,mit kleinen Schtickers darauf“, „Tropical Edition“ (Präauer 2016: 21), und Zindi, das Lieblings-Call Girl, für das er das Kreditkartenlimit seiner Mutter überzieht, kommt aus dem Balkan, hat gefärbtes Haar und „buntes Spielzeug“ in den Händen, sonst „durchquert ein schwarzer Balken ihr Gesicht“ (Präauer 2016: 57). Schimmi benutzt für sie den Ausdruck ,po-po-poetical“ (Präauer 2016: 57). Markennamen finden sich zur Genüge, nur erscheinen sie stets in Schimmis Verdrehungen: Der Vater telefoniert mit einem Nikia-Handy, aus Victoria's Secret wird Viktorias Sigrid, die Mutter trägt eine Gitschi-Tasche, etc. Hier wird nicht archiviert, sondern verballhornt und vor allem: nachgeäfft. Präauer lässt Schimmi die Kulturenzyklopädie aufrufen, bloß nicht mit dem Fokus auf Dietrich Diederichsen und Max Goldt, sondern auf Affigkeit. Tarzan, King-Kong, Ape-Man (eine Affen-Figur aus einem Superheldencomic), auch Herr Nilsson sind die Referenzfiguren, musikalisch wird der Rhythmus durch schwarzen Rap und Hip-Hop vorgegeben, erwähnt wird auch der deutsche Hip-Hopper Peter Fox mit seinem erfolgreichen Album Stadtaffe von 2008. 
Die Sprache des Romans führt fast exemplarisch das Verfahren des Samplings vor-diverse kulturelle Querverweise werden recht wild zusammengesetzt und gemixt. Zitate von Muhammad Ali - „Schwebe wie ein Schmetterling, stich wie eine Biene!“ (Präauer 2016: 176) - tönen zur Begleitung von Dschungelmusik, Jane Goodall, die Schimpansenforscherin, wird als Gegenteil der strengen Mutter imaginiert, das Callgirl Zindi erscheint als ein Inbegriff von the american way of life - ,All-american! Wie Pancakes mit Heidelbeeren, Cream und Mandelsirup zum Frühstück, wie Toastbrot mit Rührei und Speck [...], wie Burger zu Mittag mit Pommes und Fiffi Cola“ (Präauer 2016: 157) - idolisiert wird Duke Kahanamoku, der Pionier des Surfings. Schimmi denkt und spricht in chaotisch durcheinandergewirbelten urbanen Slangs, ins Auge stechen Ausdrücke, die auf Rassendifferenzen im Stadtdschungel verweisen: „Jungle fever" für sexuelle Fetischisierung der Schwarzen, „White Trash“ für weiße Unterschicht, „Gringo“, wie sich Schimmi in Gedanken an die mexikanische Putzfrau wendet, die übrigens eine Jogginghose mit der Aufschrift „La Fuerza del Destino“ trägt (Präauer 2016: 82), etc. Die Rassendifferenzen versteht Schimmi nicht bzw. er nimmt sie als gegeben hin, zentral sind aus seiner Sicht die Gattungsdifferenzen: Er selbst liebäugelt mit der Vorstellung, sein Name komme eigentlich von „Schimpanse“ (Präauer 2016: 183), aus seiner emphatischen Affirmation der Affen heraus ${ }^{9}$ kommentiert er die menschliche Welt mit einer Mischung aus Verwunderung: „Der Dschungel wiederum regelt alles selbst. Aber die Siffilisation?“ (Präauer 2016: 98) und Resignation, mehrmals wiederholt er: „Realismus, Baby“ (Präauer 2016: 116 u. 131).

Wie auch in Flors Roman ist es nicht die Figur selbst, die ihre ironische Sicht auf die Wirklichkeit mitteilt; die Ironie ergibt sich bei Präauer weniger aus der Form des Romans bzw. aus der diskursiven Bricolage als aus den Diskrepanzen, die in der kompulsiv vorgetragenen, naiv-hellsichtigen Rede Schimmis sichtbar sind. Ein viriler „schwarzer Gorilla“ erobert den City-Jungle (klaut Klamotten und ein BMX-Fahrrad), auf der Suche nach Girls und „Sexualismus“, und es geschieht in einer Sprache, die zum einen die kulturenzyklopädischen Einträge aus dem Bereich der Abenteuerfilme und -comics (Tarzan, King Kong) aufruft, zum anderen das urbane Wörterbuch der schwarzen Pop-Kultur (Hip-Hopper und Rapper, Boxer, Surfer) zitiert. Die Schieflage, die durch so suggerierte Ähnlichkeit zwischen den Schwarzen und dem Affen entsteht, ist wohl einkalkuliert - als provokativ-selbstironische Selbstbezeichnung schwarzer Rapper ${ }^{10}$ erzeugt sie im Buch einer weißen Autorin den Effekt von Political Incorrectness, mit dem Pop bekanntlich gern spielte. ${ }^{11}$ (Man denke etwa an den Namen „Neger Negersen“, den Rainald Goetz für Diederichsen erfand) (Goetz 1986: 19 u. 24) Präauers

9 „Man tut den Affen auch unrecht, wenn man ihnen menschliche Eigenschaften zuschreiben wollte“ (Präauer 2016: 143); ,Sie sind der Natur so entfremdet, dass sie beim Anblick eines Affen im Supermarkt gleich an Überfall denken.“ (Präauer 2016: 185)

${ }^{10}$ Vgl. etwa das Album des Rappers Sean Price Monkey Barz von 2005.

${ }^{11}$ Vgl. Christoph Rauen: „Pop senkt die Entmutigungsschwelle für ,respektlose, naseweise, plumpe und grelle 'Sprechakte, die im Rahmen pragmatischer Rede unwahrscheinlich wären, aber durch ,Entpflichtung 
Ironie ist genussvoll, weil sie im kulturpessimistischen Abgrund nicht versinkt - die Affenverkleidung eröffnet mehrere Möglichkeiten, vor allem aber wirkt sie wie ein Spiegel, in dem sich die „Siffilisation“" mit ihren Gesetzen der „Kissalität", „,Kirrelation" und „Affizienz" wiedererkennen kann. ${ }^{12}$ Das Affenkostüm ,entpflichtet die Rede"13 und macht jegliche ideologiekritischen Pointierungen überflüssig, vor allem wenn sie - hier wieder in Pop-Manier - kategorisch verkündet werden: „Der Dschungel ist überall.“ (Präauer 2016: 202)

\section{Ironie postmodern: Cordula Simons Wie man schlafen soll}

Hayden White hat in seinem vielzitierten Buch Auch Klio dichtet Ironie als die rhetorische Struktur des Postmodernismus bezeichnet (White 1986), für die postmoderne Literatur wurde der Gestus der Ironie mehrfach als kennzeichnend, ja sogar als ,zentrales stilistisches Merkmal“ (Birnstiel, Schilling 2012: 84) genannt. Die Postmoderne

[...] unterläuft Darstellungs- und Gattungskonventionen hergebrachter Literatur ebenso wie inhaltliche und formale Lesererwartungen, sie depotenziert die normativen Ansprüche der literarischen Überlieferung und die erzieherischen Postulate einer älteren Ästhetik. Aussagen früherer Texte werden durch ironische Zitation negiert; über die Verständigung auf den Modus der Ironie als Sagweise ist auch die Möglichkeit gegeben, im ironischen Sprechen ernstgemeinte Aussagen zu treffen, ohne dass diese ,naiv' klingen. (Birnstiel, Schilling 2012: 84)

Cordula Simons Roman Wie man schlafen soll von 2016 spielt in einer nicht näher präzisierten Zukunft in einer Stadt namens Lightraff, die am Meer liegt und in der es ständig regnet. Die Feuchtigkeit stört nicht, solange man Arbeit hat, und diese verlieren die drei Figuren - Haye, Schreiber und Koslov - aus ungewissen Gründen schon im ersten Teil des Romans. In der Stadt lebt man, weil „auf dem Lande nichts mehr zu holen“" (Simon 2016: 29) ist, wegen Hitze und Dürreperioden. Lightraff ist eine künstliche Stadt, die immerhin noch imstande ist, Wasserversorgung, Arbeits- und Schlafplätze zu garantieren. ${ }^{14}$ So zumindest die Werbeversprechen der „Firma“, die

des Sprechers von der Gültigkeit seiner Aussagen problemlos möglich sind." Rauen, Ch. (2010). Pop und Ironie. Popdiskurs und Popliteratur um 1980 und 2000. Berlin, New York: de Gruyter, S. 46.

${ }^{12}$ Oh Schimmi weist in der Zivilisationskritik einige Ähnlichkeiten mit dem Roman Ishmael von Daniel Quinn (1992) auf, in dem der titelgebende Ishmael ein telepathiebegabter Gorilla ist, der als Lehrer eines nach Sinn suchenden Jungen fungiert.

${ }^{13}$ Vgl. Karl Eibl: „Generell kann man sagen: Die entpflichtete Rede ermöglicht es, über Dinge zu reden, über die man - aus den verschiedensten, trivialen wie erhabenen Gründen - eigentlich nicht reden kann oder darf oder soll, über die man aber trotzdem reden will oder soll oder muß." Eibl, K. (2004). Animal poeta. Bausteine der biologischen Kultur- und Literaturtheorie. Paderborn: mentis, S. 346.

14 „Hier hatte man eine Zukunft. Lightraff war der Ausweg. Lightraff bedeutete, nicht mehr ständig auf den Tod warten zu müssen. Lightraff war ein Licht am Ende des langen Eisennbahntunnels gewesen [...]“ (Simon 2016: 42). 
sich allmählich als Trug erweisen, was die Figuren daran zu spüren bekommen, dass sie mit Arbeitsverlust die Lightraffkarte und damit den Zugang zur Straßenbahn, zur Wohnung und zur Bibliothek verlieren. Keine Straßenbahn und keine Bibliothek erscheinen allerdings als Randprobleme angesichts der wichtigsten Lebensfunktion der Lightraffer - dem Schlafen. Arbeit wird schichtenweise organisiert - wer sein Pensum Arbeit erledigt, darf schlafen. Daher können sich die drei Figuren eine Wohnung und ein Bett teilen - nach acht Stunden Schlaf wird gewechselt, was auch perfekt funktioniert, solange alle Arbeit haben. Schlafendürfen scheint die einzige Motivation zu sein, eine Arbeitsstelle zu behalten - denn wenn auch zwei von den drei männlichen Figuren Freundinnen haben (der dritte Koslov, hat eine Lieblingsbar), gibt es außer der Arbeit und dem Schlaf nichts, was von Bedeutung wäre. Wenn Runné, Hayes Arbeitskollege, im Büro am Schreibtisch tot aufgefunden wird, entdeckt man, dass er in den Schichtpausen im Büroschrank schlief (Simon 2016: 98).

In Simons Zukunftswelt ist man offensichtlich nicht so weit, dass man ,vorschlafen“ bzw. „schlaf speichern“ (Röggla 2004: 21) bzw. übertragen könnte, auch „schlafbanken“ (Röggla 2004: 22) gibt es nicht - ja, man hätte dort kein Verständnis für die Fantasievisionen des IT-Supporters aus Kathrin Rögglas wir schlafen nicht. Denn, auch wenn in Lightraff der Jargon der Optimierung nachhallt - Lightraff sei ,eine Familie“ (Simon 2016: 139), zu der man gehören will, die Firma organisiert die gesamte Stadtverwaltung - haben sich die Prioritäten gewaltig verschoben. In Rögglas protokollierten Bekenntnissen wird offen zugegeben, dass Schlaf gelegentlich im Büro zwischendurch nachgeholt wird, ${ }^{15}$ aber als Leistung gilt Schlafreduktion: „nee, schlafen sei nicht schick, [...] wer schlafe, sei auch schlecht beraten“" (Röggla 2004: 35). Es gibt welche, die ihren Posten kaum noch verlassen: ,ja, aber der herr gehringer, der arbeitet nur noch. Den sieht man immer nur im büro [...] man weiß nicht, geht er überhaupt noch nach hause?“ (Röggla 2004: 133) Während Röggla Einblicke in die Kommunikationsstrukturen, Hierarchien und Selbstoptimierungsstrategien bietet, und zwar im originellen Sound, haben die Figuren bei Simon eine Sorge - wo schlafe ich, wo ich doch ohne Arbeit kein Recht auf ein Bett habe?

Simons Roman lässt sich wie eine ironische Fortsetzung von Rögglas Text im Genre der Dystopie lesen - die Logik der potenzierten Arbeitswelten, die schon bei Röggla auf eine Suchtspirale mit Selbstentfremdung an durchstandardisierten Nicht-Orten hinauslief, wird bei Simon in einen Gesamtkontext der Zivilisationsentwicklung zu Ende gedacht. Dass Simon dem Aspekt von Arbeit und Schlaf mit Ironie begegnet, merkt man allein an der Figur der Übertreibung - die Optimierung des Arbeitseinsatzes geht in Lightraff auf Kosten der Privatheit und der Intimsphäre der Beschäftigten. Wenn Schreiber entlassen wird, erfährt er, er bekomme die letzte Lohnauszahlung in flüssiger Gelatine ausgezahlt, die übrigens angesichts der Entwertung der Lightraff-Währung zu

15 „Und wenn tage superheftig waren, hat sie sich manchmal in irgendein büro zurückgezogen und nur kurz zehn, fünfzehn minuten die augen zugemacht. - jeder kennt das doch.“ (Röggla 2004: 21) 
einem begehrten Zahlungsmittel wird. Der einzige, der die heimkehrenden Schichtarbeiter an der Wohnungstür begrüßt, ist Rob, der autonome Staubsauger.

Simons Ironie lässt sich auch erkennen, wenn man auf die Aspekte des Wohnens, Arbeitens und Schlafens einen historischen Blick wirft - Lightraff erscheint aus dieser Perspektive wie eine (Arbeiter)Stadt der Zukunft und der Vergangenheit, zugleich industriell und postindustriell. Die urbane Wohnungsnot und die damit zusammenhängende Prekarität der Lebensverhältnisse, also auch des Schlafens, sind in die Geschichte der Industrialisierung fest eingeschrieben. Es reicht ein Blick auf die Lage der Industriearbeiter in den ersten Jahrzehnten des 20. Jahrhunderts, um die Zusammenhänge zwischen Klassenzugehörigkeit und der durch Wohnmangel bedingten Schlaflosigkeit zu beleuchten - der Begriff „Schlafgänger“, der seit der zweiten Hälfte des 19. Jahrhunderts alleinstehende Schichtarbeiter ohne eigenen Wohnraum bezeichnete (Brüggemeier/ Niethammer 1978), könnte ohne weiteres auf Simons Figuren angewendet werden. Lightraff trägt somit Züge der Beschäftigungs- und Wohnpolitik in Großkonzernen der industriellen Ära (etwa: Thyssen) wie auch den postindustriellen globalen Corporations - es garantiert die Versorgung der Mitarbeiter mit Wohnraum und Zugang zu Institutionen und Dienstleistungen wie die Mensa, die Bibliothek, der Chatroom (nur für Verheiratete gratis) - und zeigt zugleich alle Merkmale einer postindustriellen $\mathrm{Zu}$ kunftsstadt, denn im Roman heißt es, es gäbe keine anderen Lebensorte mehr. ${ }^{16}$ Das Klassenbewusstsein spielt zwar bei Simon nur am Rand eine Rolle, aber die (bittere) Ironie der dystopischen Konstruktion macht deutlich, dass die scheinbare Klassenlosigkeit im Grund keinen Fortschritt gegenüber der Arbeitsrealität von vor hundert Jahren bedeutet - denn in der Lightraff-Gesellschaft bleibt die Hierarchie zwischen Reichen und Armen, und damit die Abhängigkeit der Letzteren, bestehen, ja sie ist sogar wegen der katastrophalen Versorgungslage außerhalb der Stadt absolut.

In Rögglas wir schlafen nicht konnte man eine ironische Note u. a. daran erkennen, wie die Autorin die Zitatmontage organisierte - so wurde die Widersprüchlichkeit der Aussagen bzw. die Peinlichkeit der Absicherungsstrategien und der „durchhalteparolen“ (Röggla 2004: 189) sichtbar. Simon zieht aus der konstruktiven Ironie Rögglas radikale Schlüsse, indem sie eine Diegese konstruiert, in der nicht Arbeit, sondern Arbeitslosigkeit zum Schlafverlust führt, und zwar aus rein materiellen Gründen: „Wer nicht arbeitet, darf auch nicht schlafen" (Simon 2016: 102). Aber auch derjenige, der arbeitet, wird bei Simon zum „Schlafgänger“ - das Bett wie auch der knappe Wohnraum gehören dem Lightraff; was vor hundert Jahren notbedingt durch zwischenmenschliche Arrangements geregelt wurde, ${ }^{17}$ erledigen nun Computer und andere Maschinen. Ge-

${ }^{16}$ „Wir können nicht zurück aufs Land. Dort gibt es nichts mehr. Nicht für uns. Nicht für sonst jemanden. Nichts wächst mehr. [...] Umweltgifte, hat der Spezialist gesagt. [...] Nichts wächst. Die Tiere sind alle tot.. [...] Alles was gewachsen ist, war giftig. Und jetzt ist der Boden tot. [...] Die Menschen verschwinden. [...] Das Wasser ist grenzwertig.“ (Simon 2016: 149)

${ }^{17}$ Vgl. J. Ronald Shearer: „Married miners with their own dwellings often let a small room, or roof loft, or even simply a bad in a corner to single miners in order to augment the family income. These lod- 
meinsames Wohnen der drei Schlafgänger-Figuren erzeugt kein Gemeinschaftsgefühl: „Koslov, Haye und Schreiber empfanden sich nicht als Mitbewohner. Sie empfanden sich als Personen, die zufällig Bett, Kloschüssel und Tricof teilten, aber nichts weiter“ (Simon 2016: 102). ${ }^{18}$

Den Hinweis darauf, dass Simons Roman auf Rögglas Roman rekurriert, könnte man außerdem am Verfahren des mise en abyme erkennen - zweimal entspannt sich bei Simon eine selbstreferentielle Schleife, eine Figur findet in der Bibliothek zufällig das Buch Wie man schlafen soll und liest kurze Passagen aus ihm, die sich später identisch in der Diegese wiederholen und zum Plot gehören (Simon 2016: 74 u. 190). Mise en abyme, beliebt bei Autor_innen des Nouveau Roman wie auch der Postmoderne, ,überschreitet fast die Grenze zur Identität" (Genette 2010: 151), d. h. nivelliert de facto die narrative Distanz, sie erzeugt in einem Narrativ den Effekt des ,freien Falls“ , 19 den Verlust von Orientierung. Durch dieses Element erreicht die Ironie bei Simon den Zustand, den Paul de Man als „unrelieved vertige, dizziness to the point of madness“ bezeichnet hat (de Man 1969: 198) - Koslov, die Figur, die die Entdeckung macht, dass alles, was sie erlebt, schon im Buch Wie man schlafen soll enthalten ist, stirbt nach dem Satz: „So etwas wie nur lesen gibt es nicht“" (Simon 2016: 190).

In der narrativen Spiegelung, die mise en abyme erzeugt, setzt Simon ein wichtiges Signal, nämlich positioniert den Standpunkt des Subjekts und des Objekts der Ironie auf einer Ebene. „So etwas wie nur lesen gibt es nicht“ - das Gelesene bleibt nicht ohne Folgen, lesend wird man/frau zum Mitwissenden und Mitverantwortlichen. Auch Präauer lässt jegliche Hierarchisierung fallen, wenn sie Schimmi die „Siffilisation" kommentieren und die Überlegenheit der menschlichen Spezies relativieren lässt. Flor rekapituliert die gegenwärtigen Netz-Verhalten und Diskurse gefiltert durch das Bewusstsein des minderjährigen Models, dabei setzt sie ein Foto mit einer unendlichen Spiegelreihe zu einem Blog-Eintrag ein, wo es um Darmspiegelung geht. Damit wird auch die romantische Metapher der Universalpoesie und ihrer Potenzierung ,in einer endlosen Reihe von Spiegeln“20 evoziert, ironisch allerdings, weil sie den potenziell

gers were considered almost a part of the family and enjoyed meals or even washing and sewing services from the wife." Shearer, J.R. (1999). Shelter from the Storm: Politics, Production and the Housing Crisis in the Ruhr Coal Fields, 1918-1924. Journal of Contemporary History, vol. 34, no. 1, S. 19-47, hier S. 29.

${ }^{18}$ Tricof ist im Roman eine Art Hauscomputer, der die Vorgänge in der Wohnung registriert und die Kommunikation zwischen den Bewohnern gewährleistet (zumindest solange er funktionsfähig ist).

${ }^{19} \mathrm{Vgl}$. Berel Lang: „How much more daring - how much more truly ironic - the free-fall promised by skeptical irony, the mise en abyme that offers no footholds or handholds, no stopping place in or out of consciousness (itself a mystifying artefact), and where difference, the prime ingredient of irony, is prior to identity.“ Lang, B. (1996). The Limits of Irony. New Literary History, vol. 27, no. 3, S. 571-588, hier S. 577.

${ }^{20} \mathrm{Vgl}$. Friedrich Schlegel: „Und doch kann auch sie [die romantische Poesie] am meisten zwischen dem Dargestellten und dem Darstellenden, frei von allem realen und idealen Interesse auf den Flügeln der poetischen Reflexion in der Mitte schweben, diese Reflexion immer wieder potenzieren und wie in einer endlosen Reihe von Spiegeln vervielfachen." Schlegel, F. (2007). 116. Athenäum-Fragment. In F. Schlegel, 
unendlichen Prozess der Innen- und Außenschau der poetischen Reflexion auf die Körperlichkeit des Models projiziert (Abb. 5).

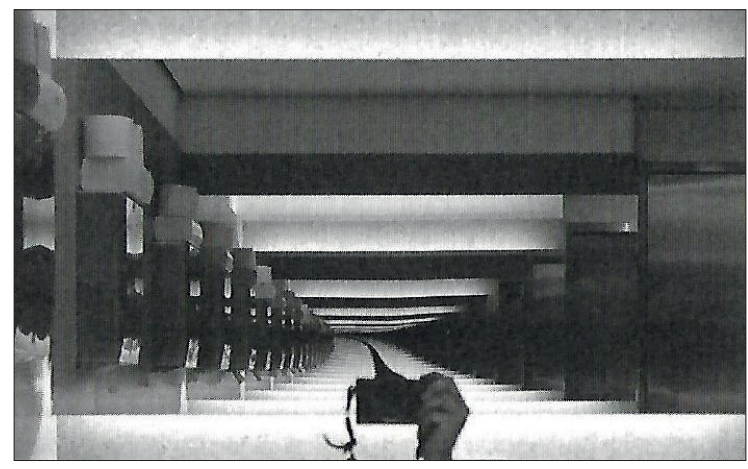

Abb. 5.: Ich in Gelb, S. 115.

\section{Fazit}

Drei sehr unterschiedliche Romane von drei österreichischen Autorinnen mit unterschiedlichen generationellen Prägungen (Flor ist Jahrgang 1968; Präauer 1979; Simon 1986) setzen jeweils ein anderes Ironie-Konzept ein (und es ist übrigens die Digital Naive Flor, die einen Blog-Roman verfasste). Man könnte es als Bestätigung der anfangs zitierten These von Röggla betrachten, der zufolge Ironie in Österreich Tradition habe; aber die These ließe sich ergänzen: Das Fortschreiben der österreichischen Ironie-Tradition bedeutet heutzutage, dass die literarischen Texte sich diverse Ironie-Konzepte einverleiben und von der Pluralität dieser profitieren. Kennzeichnend ist dabei, was die so praktizierte „politics of irony“ zustande bringt, nämlich eine unterschiedlich realisierte Rekapitulation laufender Debatten, ob es sich um Digital Memory und Digital Identity, Post-Anthropozän und die Neuentdeckung des Tiers durch die Human-Animal-Studies oder Neoliberalismus und Umstrukturierung der Arbeitswelt handelt.

Schriften zur kritischen Philosophie 1795-1805. Hrsg. v. Andreas Arndt. Hamburg: Meiner, S. 69. Siehe auch: Douglas C. Muecke: „Ironist and victim being thus on the same level, we find new 'horizontal' images of irony designed to express the newly perceived elements of equivocation, paradox, ambiguity, doubt and other such dualities. One such image is that of the ambiguous drawing, for example, the solid cube that transforms itself, while we look, into a hollow box. Another more, frequently encountered, is the mirror or even the hall of mirrors that multiplies reflections to infinity (and one does not that "reflections" is also a metaphor for the mode has itself for its own object)." Muecke, D.C. (1983). Images of Irony. Poetics Today, vol. 4, no. 3, S. 399-413, hier S. 407f. 


\section{Literatur}

Assmann, A. (2018). Über Erinnerung und Wahrheit. Medien und Öffentlichkeit. Heidelberger Akademie der Wissenschaften. Jahrbuch, 122-129.

Baßler, M. (2005). Der deutsche Pop-Roman. Die neuen Archivisten. München: C.H. Beck.

Birnstiel, K. \& Schilling, E. (2012). Einleitung. Postmodernes Erzählen und Konsequenzen für die Theorie. In K. Birnstiel \& E. Schilling (Hrsg.), Literatur und Theorie seit der Postmoderne (S. 83-91). Stuttgart: Hirzel.

Booth, W.C. (1975). A Rhetoric of Irony. Chicago, London: University of Chicago Press.

Brüggemeier, F.J. \& Niethammer, L. (1978). Schlafgänger, Schnapskasinos und schwerindustrielle Kolonie. In: J. Reulecke, W. Weber (Hrsg.), Fabrik, Familie, Feierabend (S. 135-176). Wuppertal: Hammer.

Eibl, K. (2004). Animal poeta. Bausteine der biologischen Kultur- und Literaturtheorie. Paderborn: mentis.

Flor, O. (2015). Ich in Gelb. Salzburg, Wien: Jung und Jung.

Flor, O. (23. Juni 2015) im Gespräch mit Katrin Hillgruber. „Wer sagt hier „Ich“? Und in welcher Absicht? Abgerufen von http://volltext.net/texte/wer-sagt-hier-ich-und-in-welcher-absicht/.

Freud, S. (1940). Das Medusenhaupt. Internationale Zeitschrift für Psychoanalyse und Imago, Bd. XXV, H. 2, 273-274.

Gauhofer, K. (20 Oktober 2012). Kathrin Röggla: „Enge der Heimat ist nichts für mich.“ Die Presse [online]. Abgerufen von https://diepresse.com/home/schaufenster/salon/1303644/Kathrin-Roeggla_Enge-derHeimat-ist-nichts-fuer-mich.

Genette, G. (2010). Die Erzählung (3. Aufl.) (A. Knop, Übers.). München: Fink.

Goetz, R. (1986). Irre. Frankfurt a. M.: Suhrkamp.

Herbst, A.N. (2005). Das Weblog als Dichtung. Einige Thesen zu einer möglichen Poetologie des Weblogs. Edition taberna kritika. Abgerufen von http://www.etkbooks.com/wp-content/uploads/etkcontext04_spa5_anh_weblog.pdf.

Hoskins, A. (2011). 7/7 and Connective Memory: Interactional Trajectories of remembering in post-scarcity culture. Memory Studies, 4 (3), 269-280.

Hoskins, A. (2017). The Restless Past: An Introduction to Digital Memory and Media. In A. Hoskins (Hrsg.), Digital Memory and Media: Media Pasts in Transition (S. 1-24). New York: Routledge.

Hutcheon, L. (1994). Risky business: The «Transideological» Politics of Irony. London: Routledge.

Japp, U. (1983). Theorie der Ironie. Frankfurt a. M.: Klostermann.

Lang, B. (1996). The Limits of Irony. New Literary History, vol. 27, no. 3, 571-588.

de Man, P. (1969). The Rhetoric of Temporality. In Ch.S. Singleton (Hrsg.), Interpretation: Theory and Practice (S. 173-210). Baltimore: Johns Hopkins Press.

Martens, R. (29 Juni 2015). Wenn Google löschen soll. Hamburger Mediensymposium zum Thema „Konflikte auf digitalen Plattformen.“ Medienkorrespondenz. Abgerufen von https://www.medienkorrespondenz.de/leitartikel/artikel/wenn-google-loeschen-soll.html.

Moser, W. (1984). The Factual in Fiction: The Case of Robert Musil. Poetics Today, vol. 5, no. 2, 411-428.

Muecke, D.C. (1983). Images of Irony. Poetics Today, vol. 4, no. 3, 399-413.

Musil, R. (1978). Der Mann ohne Eigenschaften. Gesammelte Werke. Hrsg. v. A. Frise. Reinbek bei Hamburg: Rowohlt.

Präauer, T. (2016). Oh Schimmi. Göttingen: Wallstein.

Rauen, Ch. (2010). Pop und Ironie. Popdiskurs und Popliteratur um 1980 und 2000. Berlin, New York: de Gruyter.

Rohde-Dachser, Ch. (1991). Expedition in den dunklen Kontinent. Weiblichkeit im Diskurs der Psychoanalyse. Berlin, Heidelberg: Springer.

Röggla, K. (2004). wir schlafen nicht. Frankfurt a. M.: Fischer. 
Schlegel, F. (2007). 116. Athenäum-Fragment. In F. Schlegel, Schriften zur kritischen Philosophie 17951805. Hrsg. v. A. Arndt. Hamburg: Meiner.

Shearer, J.R. (1999). Shelter from the Storm: Politics, Production and the Housing Crisis in the Ruhr Coal Fields, 1918-1924. Journal of Contemporary History, vol. 34, no. 1, 19-47.

Simon, C. (2016). Wie man schlafen soll. Salzburg, Wien: Residenz.

Terdiman, R. (1985). Discourse / Counter-Discourse: A Theory and Practice of Symbolic Resistance of Nineteenth-Century France. Ithaca, New York, London: Cornell University Press.

White, H. (1986). Auch Klio dichtet oder Die Fiktion des Faktischen. Studien zur Tropologie des historischen Diskurses (B. Brinkmann-Siepmann u. Th. Siepmann, Übers.). Stuttgart: Klett-Cotta. 